\title{
Prefrontal/Amygdalar System Determines Stress Coping Behavior Through 5-HT/GABA Connection
}

\author{
Diego Andolina*, I,2, Dario Maran ${ }^{3}$, Alessandro Valzania ${ }^{1,3}$, David Conversi ${ }^{1,3}$ and Stefano Puglisi-Allegra ${ }^{1,3}$ \\ 'Santa Lucia Foundation, Rome, Italy; 'Dipartimento di Scienze Cliniche Applicate e Biotecnologie, Università degli Studi dell'Aquila, Via Vetoio, \\ L'Aquila, Italy; 'Dipartimento di Psicologia and Centro 'Daniel Bovet,' Università degli Studi di Roma 'La Sapienza', Rome, Italy
}

\begin{abstract}
Coping is defined as the behavioral and physiological effort made to master stressful situations. The ability to cope with stress leads either to healthy or to pathogenic outcomes. The medial prefrontal cortex (mpFC) and amygdala are acknowledged as having a major role in stress-related behaviors, and $\mathrm{mpFC}$ has a critical role in the regulation of amygdala-mediated arousal in response to emotionally salient stimuli. Prefrontal cortical serotonin (5-hydroxytryptamine (5-HT)) is involved in corticolimbic circuitry, and GABA has a major role in amygdala functioning. Here, using mice, it was assessed whether amygdalar GABA regulation by prefrontal 5- $\mathrm{HT}$ is involved in processing stressful experiences and in determining coping outcomes. First (experiment I), bilateral selective 5-HT depletion in mpFC of mice reduced GABA release induced by stress in basolateral amygdala (BLA) and passive coping in the Forced Swimming Test (FST) (experiment 2). Moreover, prefrontal-amygdala disconnection procedure that combined a selective unilateral 5-HT depletion of mpFC and infusion of an inhibitor of GABA synthesis into the contralateral BLA, thereby to disrupt prefrontal-amygdalar serial connectivity bilaterally, showed that disconnection selectively decreases immobility in the FST. These results point to prefrontal/amygdala connectivity mediated by $5-\mathrm{HT}$ and GABA transmission as a critical neural mechanism in stress-induced behavior.

Neuropsychopharmacology (2013) 38, 2057-2067; do::I0.1038/npp.2013.107; published online 5 June 2013
\end{abstract}

Keywords: coping; corticolimbic; disconnection; GABA; serotonin

\section{INTRODUCTION}

The impact of stressors is determined by the ability of the organism to cope with the situation (Ursin and Olff, 1995). Coping consists of the behavioral and physiological efforts to master the situation (Koolhaas et al, 2007; Wechsler, 1995; Lazarus, 1966). Indeed, a stressful experience cannot be sustained at length (McEwen, 2007), and the organism thus needs to develop effective coping strategies, which may prove to be either healthy or pathogenic (McEwen, 2007; Maier and Watkins, 2010; Taylor and Stanton, 2007). Successful coping depends largely on the controllability and predictability of the stressor (Cabib and Puglisi-Allegra, 2012). As shown by human and animal studies, whenever environmental stressors are too demanding and the individual cannot cope, health is endangered. Preclinical research using animal models aimed at gaining insight into stress-related disease has now started to exploit phenotypic characterization and differential response patterns under stressful experimental conditions. Stress-associated adaptive changes may serve to increase the resistance to

\footnotetext{
* Correspondence: Dr D Andolina, Dipartimento di Scienze Cliniche Applicate e Biotecnologie Università degli Studi dell'Aquila, Via Vetoio, L'Aquila 670I0, Italy, Tel: +39 862 433523, Fax: +39 862433524 , E-mail: diego.andolina@gmail.com

Received 29 December 2012; revised 20 April 2013; accepted 22 April 2013; accepted article preview online 2 May 2013
}

pathogenic outcomes of subsequent stressful experiences, thus favoring resilience, or they may establish dysfunctional coping strategies that increase 'allostatic load' (Maier and Watkins, 2010; Maier and Seligman, 1976; Anisman et al, 1983; McEwen, 2001).

Because cortical and limbic systems have a major role in stress appraisal and adaptation, the identification of stress responses by these systems could lead to the discovery of the mechanisms responsible for healthy and pathogenic outcomes of stressful experiences. Various studies have highlighted a major role for medial prefrontal cortex (mpFC) and amygdala in stress-related behaviors (Wellman et al, 2007). The mpFC is thought to have a critical role in regulating amygdala-mediated arousal in response to emotionally salient stimuli, and the 5-hydroxytryptamine (5-HT) system within the mpFC represents a potential molecular mechanism through which the mpFC modulates corticolimbic circuitry (Fisher et al, 2009). Moreover, manipulation of GABAergic transmission within the basolateral amygdala (BLA) has a critical influence both on behavioral and emotional sequelae resulting from stress (Martijena et al, 2002; Ebner et al, 2005). This suggests that 5 -HT transmission in the mpFC is likely to engage GABA in the amygdala in stressful situations in order to determine coping outcomes. Here, using mice, it was attempted to assess whether amygdalar GABA regulation by prefrontal cortical $5-\mathrm{HT}$ is a common neural substrate involved in processing stressful 
experiences and in determining active or passive coping behavior during forced swimming (Ebner et al, 2005, 2008; Singewald et al, 2011). In particular, it was investigated whether 5-HT in prefrontal cortex is involved in brain response to uncontrollable stress through its modulating action on GABA in amygdala and whether these brain areas are part of a neural system necessary for the implementation of adaptive coping strategies. To this end, in experiment 1 , the effects of acute stressor (restraint) on mpFC 5-HT and BLA GABA release by intracerebral microdialysis were first assessed. To determine whether 5HT prefrontal transmission governs increased BLA GABA output induced by acute restraint, also the effects of selective 5-HT depletion in mpFC on GABA release in BLA were evaluated. Then, in experiment 2, to determine whether 5-HT prefrontal transmission and GABA transmission in the BLA modulates stress coping behavior, the effects of mpFC 5-HT depletion on behavior outcome in the Forced Swimming Test (FST) were evaluated. Moreover, to evaluate if mpFC 5-HT transmission modulates the activation of specific amygdala nuclei in response to stressful experience, c-fos activation was assessed in the central amygdala (CeA) and in BLA nuclei following FST as an indicator of neuronal activation (Hughes and Dragunow, 1995). In fact, induction of this immediate early gene is used extensively to assess neuronal activation following stressful stimuli (Cullinan et al, 1995; Chowdhury et al, 2000; Day et al, 2005). To assess the role of the prefrontal 5-HT/ amygdalar GABA system in controlling stress-induced behavior in FST, an asymmetric disconnection procedure was used to block the transmission of information within the specific pathways in each hemisphere.

\section{MATERIALS AND METHODS}

\section{Animals}

Male mice of the inbred C57BL/6JIco strain (Charles River Laboratories, Wilmington, MA), 8-9-week-old at the time of the experiments, were housed as previously described (Ventura et al, 2007). All experiments were conducted in accordance with Italian national law (Decreto Legislativo no. 116, 1992) governing the use of animals for research.

\section{Drugs}

Chloral hydrate, 5,7-dihydroxytryptamine (5,7-DHT), desipramine hydrochloride (DMI), L-allylglycine, and tetrodotoxin (TTX) were purchased from Sigma-Aldrich (St Louis, MO). Chloral hydrate $(350-450 \mathrm{mg} / \mathrm{kg})$ and DMI $(35 \mathrm{mg} / \mathrm{kg})$ were dissolved in saline $(0.9 \% \mathrm{NaCl})$ and injected i.p. in a volume of $10 \mathrm{ml} / \mathrm{kg}$. The 5,7-DHT was dissolved in saline containing ascorbic acid $(0.1 \%)$ and TTX $(1 \mu \mathrm{m})$, and L-allylglycine $(1 \mathrm{mM})$ was dissolved in artificial cerebrospinal fluid (CSF).

\section{Stress Protocols}

Restraint stress and FST were as described previously (Alcaro et al, 2002; Cabib and Puglisi-Allegra, 1991). Although FST is usually performed in two sessions, $24 \mathrm{~h}$ (or longer) apart, we tested animals in one testing session, because the aim was to assess coping in a stressful condition.

\section{Microdialysis}

Animals were anesthetized with chloral hydrate $(450 \mathrm{mg} /$ $\mathrm{kg}$ ), mounted in a stereotaxic frame (David Kopf Instruments, Tujunga, CA), equipped with a mouse adapter, and implanted unilaterally with a guide cannula (stainless steel, shaft outer diameter of $0.38 \mathrm{~mm}$; Metalant $\mathrm{AB}$, Stockholm, Sweden) in mpFC or BLA. The length of the guide cannula was $1 \mathrm{~mm}$ for $\mathrm{mpFC}$ and $4.5 \mathrm{~mm}$ for BLA. The guide cannula was fixed with epoxy glue, and dental cement was added for further stabilization. The coordinates from bregma (measured according to the atlas of Franklin and Paxinos, 1997; Mouse Brain Atlases 2004) were: 2.52 anteroposterior and 0.6 lateral for $\mathrm{mpFC}$ and -1.6 anteroposterior and -3.55 lateral. The probe (dialysis membrane length of $2 \mathrm{~mm}$ for $\mathrm{mpFC}$ and $1 \mathrm{~mm}$ for BLA and outer diameter of $0.24 \mathrm{~mm}, \mathrm{MAB} 4$ cuprophane microdialysis probe, Metalant $A B$ ), was introduced $24 \mathrm{~h}$ after implantation of the guide cannula. The animals were lightly anaesthetized $(350 \mathrm{mg} / \mathrm{kg})$ to facilitate manual insertion of the microdialysis probe into the guide cannula. The day before use, the membranes were tested to verify in vitro 5-HT and GABA recovery. The microdialysis probe was connected to a CMA/100 pump (Carnegie Medicine, Stockholm, Sweden), through PE-20 tubing and an ultra-low torque dual channel liquid swivel (Model 375/D/22QM, Instech Laboratories, Plymouth Meeting, PA) to allow free movement. Artificial CSF was pumped through the dialysis probe at a constant flow rate of $2 \mu \mathrm{l} / \mathrm{min}$. Experiments were carried out $22-24 \mathrm{~h}$ after probe placement as previously described (Pascucci et al, 2009). For reverse microdialysis experiments, TTX $(1 \mu \mathrm{m})$ was perfused in the BLA for $60 \mathrm{~min}$ before restraint stress, and L-allylglycine $(1 \mathrm{mM})$ was perfused in the BLA for $30 \mathrm{~min}$ before FST. In preliminary experiments, we found that L-allylglycine at this concentration is able to reduce the GABA release in BLA as does the selective 5-HT depletion in mpFC during FST, but leaving unchanged the GABA basal levels (Figure 1). Animals subjected to stress experience were put in the restraint apparatus or in the FS apparatus for 120 and $10 \mathrm{~min}$, respectivily, during microdialysis. The asymmetric disconnection procedure is based on the assumption that information is transferred serially from one structure to an efferent region, on both sides of the brain in parallel (Setlow et al, 2000; Floresco and GhodsSharifi, 2007; Belin and Everitt, 2008). The following combinations of asymmetrical bilateral conditions were used: (1) a unilateral 5-HT depletion in mpFC in combination with a contralateral inhibitor of GABA biosynthesis administration (L-allylglycine) of the BLA (disconnection), (2) a unilateral Sham depletion in $\mathrm{mpFC}$ in combination with a contralateral L-allylglycine into BLA (3) a unilateral 5 -HT depletion of the mpFC in combination with a CSF into the contralateral BLA, and (4) unilateral Sham depletion of the mpFC and CSF into controlateral BLA (Figure 2). In additional experiment, two different groups of mice implanted bilaterally in BLA and receiving either CSF or L-allylglycine were assessed in the FST. Dialysate was collected every 20 for $180 \mathrm{~min}$ for restraint condition and $10 \mathrm{~min}$ for FST. Twenty microlitres of the dialysate 
samples were analyzed by HPLC. The HPLC analysis of 5-HT concentration in the dialysates was as previously described (Pascucci et al, 2009). GABA concentrations in the dialysates were determined as described by Rea et al (2005). The detection limit of the assay was 4.2 and $0.1 \mathrm{pg}$ per $20 \mu \mathrm{l}$ (signal-to-noise ratio 2), for GABA and $5-\mathrm{HT}$, respectively.

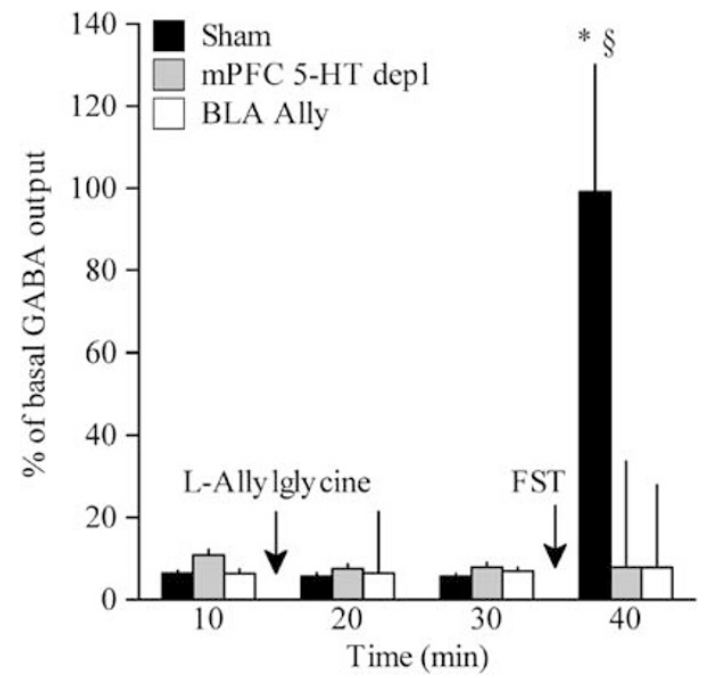

Figure I Effects of bilateral medial prefrontal cortex (mpFC) 5-hydroxytryptamine (5-HT) depletion and L-allyglycine infusion in basolateral amygdala (BLA) on GABA output in BLA exposed to 10 min of Forced Swimming Test (FST). Results are expressed as percent changes (means $\pm \mathrm{SE}$ ) from basal values preceding L-allyglycine infusion in BLA and FST. Statistical analyses were performed on raw data. $* P<0.05$ from basal values. $\$ P<0.05$ in comparison with the corresponding time point of $\mathrm{mpFC}$ Sham groups.

\section{5-HT Depletion in the mpFC}

Bilateral injection of 5,7-DHT $(2.5 \mu \mathrm{g} / 0.2 \mu \mathrm{l} / 4 \mathrm{~min}$ for each side) was made into the mpFC (coordinates: $+2.52 \mathrm{AP}$; $\pm 0.6 \mathrm{l}$; $2.0 \mathrm{~V}$ with respect to bregma (Franklin and Paxinos, 1997)), through a stainless steel cannula $(0.15 \mathrm{~mm}$ outer diameter; UNIMED, Lausanne, Switzerland), connected to a $1 \mu \mathrm{l}$ syringe by a polyethylene tube and driven by a CMA/100 pump. The cannula was left in place for an additional $2 \mathrm{~min}$ after the end of the infusion. Sham mice (Sham) were subjected to the same treatment but received intracerebral vehicle. 5-HT, norepinephrine (NE), and dopamine (DA) tissue levels in the mpFC (prelimbic (PL) or infralimbic (IL), cingulate cortex (CG), and motor cortex (Franklin and Paxinos, 1997)) were assessed to evaluate the amount and the extent of 5,7-DHT-induced depletion. Punches were obtained from brain slices (coronal sections) not thicker than $300 \mu \mathrm{m}$ (Puglisi-Allegra et al, 2000). Stainless steel tubes of 0.5 or $1.0 \mathrm{~mm}$ inside diameter were used. The coordinates were measured according to the atlas of (Franklin and Paxinos, 1997). Monoamine tissue level analysis were as described previously (Ventura et al, 2007).

Note that intracortical injection of 5,7-DHT produced a profound reduction of 5-HT tissue levels, indicating, however, a partial depletion (see results below). We use the term depletion throughout to refer conventionally to a partial depletion of the amine.

\section{Histology}

Probe placement. At the end of the experiment, mice were killed by decapitation. Brains were post-fixed in $4 \%$ paraformaldehyde, and correct probe placements were checked by visual inspection of the probe tracks on Nisslstained coronal sections $(40 \mu \mathrm{m})$. Only mice with correct probe placement in BLA and $\mathrm{mpFC}$ were considered in the results.
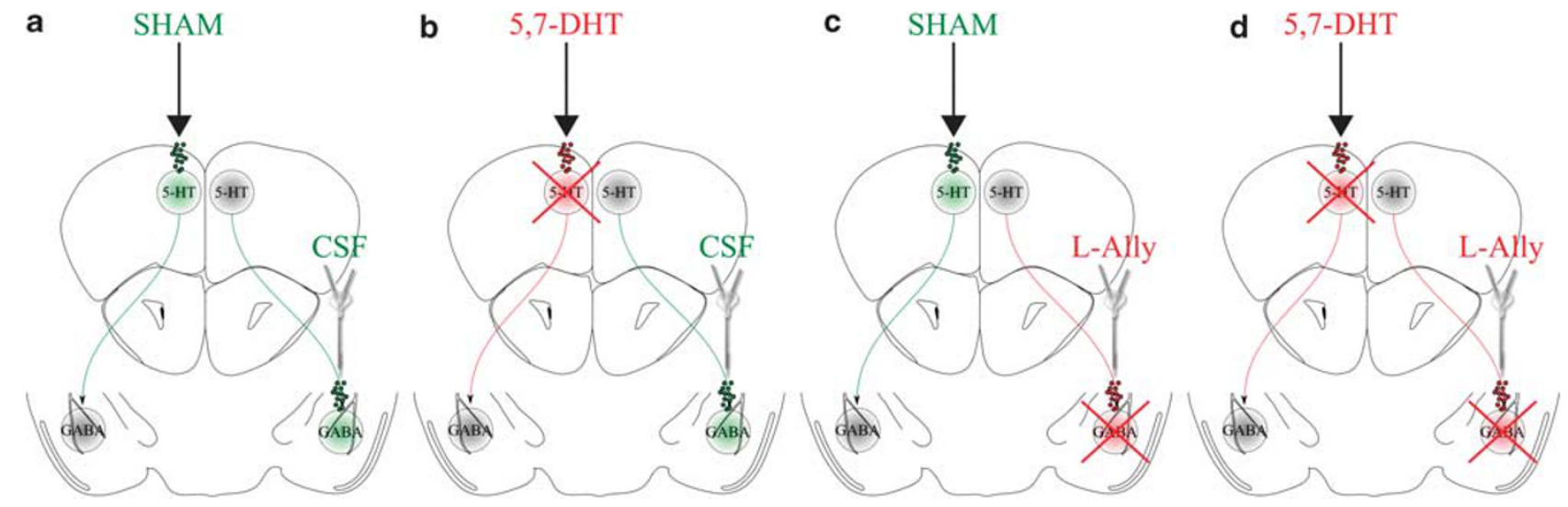

Figure 2 Schematic representation of disconnection of medial prefrontal cortex (mpFC) and basolateral amygdala (BLA) (a-d). ' $X$ ' represents the location of the unilateral inactivation to the mpFC or BLA. Green arrows represent intact pathways. Red lines and X-symbols represent blocked, nonfunctional pathways. In unilateral mpFC-5-hydroxytryptamine (5-HT)-depleted mice (red-shaded area), the mpFC relay of the pathway is lost on one side of the brain. However, on the non-depleted side (green area and arrows), the circuitry is intact and functional. When L-allyglycine (red-shaded area) is infused in the BLA contralateral to depletion, it blocks GABA output, hence impairing BLA GABA functioning in the non-5-HT-depleted side of the brain. Therefore, after unilateral L-allyglycine infusions in the contralateral BLA of mpFC-depleted animals, functional activity within the mpFC/BLA system is disrupted bilaterally. (a) This group received unilateral vehicle in mpFC (Sham) and cerebrospinal fluid (CSF) in the controlateral BLA during Forced Swimming Test (FST). (b) This group received unilateral toxin 5,7-dihydroxytryptamine (5,7-DHT) in mpFC to deplete 5-HT and CSF in the controlateral BLA during FST. (c) This group received unilateral vehicle in mpFC (Sham) and L-allyglycine (to block GABA synthesis) in the controlateral BLA during FST. (d) This group received unilateral toxin 5,7-DHT in mpFC to deplete 5-HT and L-allyglycine (to block GABA synthesis) in the controlateral BLA during FST (disconnection). 
Immunohistochemistry. One hour after the end of experiment, mice were killed by decapitation. Brains were fixed overnight in paraformaldeyde (4\%), cryoprotected in sucrose $(30 \%)$, frozen with dry ice, and cut in $40-\mu \mathrm{m}$ transversal sections. Three sections, distant approximately $-1.58,-1.70$, and $-1.82 \mathrm{~mm}$ from bregma, according to the above-mentioned atlas (Franklin and Paxinos, 1997), were immunostained for each mouse. Fos protein was detected with a rabbit polyclonal antiserum (Ab-5, oncogene, $1: 20.000)$. Secondary immunodetection was performed with biotinylated antibodies (goat anti-rabbit, $1: 1000$; Vector Laboratories, Burlingame, CA). Peroxidase labeling was performed by avidin-biotin procedure (1:1000, Vectastain ABC elite kit; Vector Laboratories). Metal-enhanced diaminobenzidine (FastDAB; Sigma) was used as the chromogen. All sections were immunostained in a single batch.

Microscopy and image analysis. Brain sections were digitally imaged with a Nikon Eclipse 80i light-transmission microscope and a Nikon DS-5M CCD camera. Image analysis was performed with public domain software ImageJ (http://rsb.info.nih.gov/ij) on a Debian system as previously described (Conversi et al, 2010). Bilateral photomicrographs of amygdala were captured with the $\times 4$ objective, stored as TIFF files and opened within ImageJ. Then, BLA and CeA were delineated with the polygon selection tool on the basis of landmarks provided by Nissl-stained adjacent sections according to the above-mentioned atlas (Franklin and Paxinos, 1997). The density ( $n$ of nuclei/ $0.01 \mathrm{~mm}^{2}$ ) of immunostained nuclei was measured using the maximum finder algorithm: (http://fiji.sc/cgibin/gitweb.cgi?p=imagej.git; $\mathrm{a}=\mathrm{blob} ; \mathrm{f}=\mathrm{ij} / \mathrm{plugin} / \mathrm{filter} /$ MaximumFinder.java).

Bilateral measures were averaged for each section and then for each rostrocaudal level obtaining a single measure for each subject.

\section{Statistics}

Forced swimming test. Statistical analyses for FST were run on the duration (second) of immobility behavior response along 10-min test. Data were analyzed by oneway analysis of variances (ANOVAs) (Sham vs bilateral 5HT depleted; bilateral CSF in BLA vs bilateral L-allyglycine in BLA). Data of the disconnection experiment were analyzed using one-way ANOVAs for independent measures for each treatment (mpFC serotonin depleted (unilateral)/ L-allyglycine in BLA (controlateral), mpFC Sham (unilateral)/ L-allyglycine in BLA (controlateral), $\mathrm{mPFC}$ serotonin depleted (unilateral)/CSF in BLA (controlateral), and mPFC Sham (unilateral)/CSF in BLA (controlateral)). Duncan's test was used post hoc; in all cases, the level of significance was set at 0.049 .

5-HT depletion in mpFC. The effects of prefrontal serotonin depletion on tissue levels of DA, NE, and 5-HT in $\mathrm{mpFC}$ were analyzed by three-way ANOVA. The factors were: treatment (two levels: Sham and 5-HT depleted) and experiment (three levels: FST experiment, microdialysis experiments, and disconnection experiment). Individual between-group comparisons were carried out when appro- priate by post hoc test, Duncan's multiple-range test. Statistical analyses were carried out on data from FST, microdialysis, and disconnection experiment.

Microdialysis. The effect of restraint stress on 5-HT release in $\mathrm{mpFC}$ and GABA release in BLA was analyzed (Sham, 5-HT-depleted; CSF, CSF + TTX) by repeatedmeasures ANOVA (time, seven level 0, 20, 40, 60, 80, 100, and 120 of restraint). The effect of TTX on GABA release in BLA during the restraint stress was analyzed by repeatedmeasures ANOVA (time, seven level 0, 20, 40, 60, 80, 100, and 120 of restraint). The effect of FST on GABA release in BLA was analyzed (mpFC Sham, mpFC 5-HT-depleted, and BLA L-allyglycine groups) by repeated-measures ANOVA (time, five level $0,10,20,30$, and 10 of FST). Statistical analyses were performed on all cases on raw data (concentration of $\mathrm{pg} / 20 \mu \mathrm{l}$ ), and data were presented in figures as percent change from baseline level. Simple effects were assessed by one-way ANOVA for each time point. Individual between-group comparisons were carried out when appropriate by post hoc test, Duncan's multiple-range test. The effects of selective 5 -HT depletion in $\mathrm{mpFC}$ and on basal extracellular GABA levels in BLA were analyzed separately in each group (Sham, 5-HT depleted). Duncan's test was used post hoc also in this case.

Immunohistochemistry. Group (Sham, 5-HT depleted) differences in c-fos density were evaluated by independent one-way ANOVAs for each brain structure (CeA and BLA), followed by Duncan's tests when allowed.

\section{RESULTS}

\section{Experiment 1}

Selective 5-HT depletion in mpFC reduces stress-induced GABA outflow in the BLA. To assess if prefrontal cortical 5-HT affects GABA in the BLA during a stressful experience, intracerebral microdialysis was used to evaluate the effects of selective 5-HT depletion in mpFC on prefrontal 5-HT and GABA output in the BLA of mice subjected to restraint stress.

Prefrontal 5,7-DHT produced a significant decrease of 5-HT tissue levels in PL (about - 84\%) and IL (about $-68 \%$ ), whereas it spared NE and DA. No significant changes in amine levels were evident in CG and the motor cortex, two areas close to PL and IL, thus showing that the effects of depletion are limited to the two medial cortical areas (Table 1).

Restraint produced a time-dependent increase of 5-HT output in mpFC and of GABA output in the BLA. Extracellular 5-HT attained a maximal increase of $\sim 150 \%$ (at 40 and $120 \mathrm{~min}$ ), whereas GABA attained a maximal increase of $\sim 200 \%$ (at $20 \mathrm{~min}$ ). Selective 5-HT depletion in mpFC canceled out the increase of both 5-HT in mpFC and GABA in BLA at all time points $(0-120 \mathrm{~min})$ but the last one $(120 \mathrm{~min})$ as far as GABA in the BLA is concerned (Figure 3). Statistical analysis revealed a significant treatment $\times$ time interaction $\left(5-\mathrm{HT}: \mathrm{F}_{6.72}=2,39 ; P<0.05\right.$ Sham group $(n=7)$, 5-HT-depleted group $(n=7)$; GABA: $\mathrm{F}_{6.84}=3,36 ; \quad P<0.01$ Sham group $(n=8), 5$-HT-depleted group $(n=8))$. Note that no significant differences were 
Table I Effects of 5,7-DHT Infusion in Medial Prefrontal Cortex on Serotonin (5-HT), Norepinephrine (NE), and Dopamine (DA) Tissue Levels (ng/g wet weight) in Different Prefrontal Cortex Areas of Sham group and 5-HT-Depleted Group

\begin{tabular}{|c|c|c|}
\hline & Sham & 5-HT depleted \\
\hline \multicolumn{3}{|c|}{ Prelimbic cortex } \\
\hline 5-HT & $775 \pm 131$ & $126 \pm 32 * * *$ \\
\hline NE & $660 \pm 58$ & $638 \pm 59$ \\
\hline DA & $117 \pm 20$ & $105 \pm 9$ \\
\hline \multicolumn{3}{|c|}{ Infralimbic cortex } \\
\hline $5-\mathrm{HT}$ & $470 \pm 39$ & $|5| \pm 26 *$ \\
\hline NE & $409 \pm 32$ & $427 \pm 68$ \\
\hline DA & $110 \pm 26$ & $124 \pm 17$ \\
\hline \multicolumn{3}{|c|}{ Cingulate cortex } \\
\hline $5-\mathrm{HT}$ & $404 \pm 37$ & $462 \pm 44$ \\
\hline $\mathrm{NE}$ & $619 \pm 40$ & $747 \pm 76$ \\
\hline DA & $100 \pm 5$ & $118 \pm 16$ \\
\hline \multicolumn{3}{|c|}{ Motor cortex } \\
\hline 5-HT & $905 \pm 113$ & $949 \pm 93$ \\
\hline NE & $513 \pm 54$ & $606 \pm 32$ \\
\hline DA & $64 \pm 6$ & $59 \pm 4$ \\
\hline
\end{tabular}

$* P<0.01 ; * * P<0.001$.

found in mpFC 5-HT and BLA GABA basal levels between the two groups (5-HT: Sham group, $0.89 \pm 0.07 \mathrm{pg} / 20 \mu \mathrm{l}$; 5-HT-depleted group, $1.02 \pm 0.03 \mathrm{pg} / 20 \mu \mathrm{l}$; GABA: Sham group $54.8 \pm 0.4 \mathrm{pg} / 20 \mu \mathrm{l}$; and 5-HT-depleted group, $66.7 \pm 0.9 \mathrm{pg} / 20 \mu \mathrm{l})$. Moreover, TTX infused through the microdialysis probe canceled out the GABA output in BLA induced by restraint stress $\left(\left(\mathrm{F}_{6,72}=3,83 ; P<0.05\right.\right.$, Sham group $(n=7)$, TTX group $(n=7))$, thus indicating that GABA results from release. The present results show that intact 5-HT transmission in mpFC is a necessary condition for the stimulation of BLA GABA release induced by stress, indicating that mpFC 5-HT transmission modulates BLA GABA release, and suggesting a role for the prefrontalamygdala system in stress response.

\section{Experiment 2}

Selective 5-HT depletion in mpFC or L-allylglycine bilateral infusion in $B L A$ reduced immobility in the FST. On the basis of results from experiment 1 showing a prefrontal 5-HT-amygdalar GABA circuitry in response to stress, it was investigated if this system is involved also in controlling coping behavior in the FST. First, the effect of bilateral prefrontal selective 5 -HT depletion in $\mathrm{mpFC}$ and L-allylglycine $(1 \mathrm{mM})$ bilateral infusion in BLA were assessed on behavioral outcomes in the FST, a well-known experimental condition commonly used to investigate stress-related behavior and to model depressive-like behavior in rodents (Porsolt et al, 1977; Valentino et al, 2010; Ebner et al, 2005). Selective bilateral 5-HT depletion in mpFC (Sham group $(n=8) 293.18 \pm 6.6 \mathrm{~s}$, 5-HT-depleted group $\left.(n=8) \quad 122.19 \pm 13.3 \mathrm{~s}, \quad \mathrm{~F}_{1.14}=132.2, \quad P<0.01\right) \quad$ or bilateral BLA L-allylglycine infusion (bilateral BLA CSF $(n=6) \quad 307.5 \pm 16.1 \mathrm{~s}$, bilateral BLA L-allylglycine group $\left.(n=7) \quad 135.2 \pm 8.6 \mathrm{~s}, \quad \mathrm{~F}_{1.11}=95.79, \quad P<0.01\right)$ significantly reduced immobility. These results show that both mpFC 5-HT and BLA GABAergic transmission control immobility in the FST.

It is worth noting that although resulting from two independent experiments, Sham group (depletion experiment) was non statistically different from Bilateral BLA CSF group, and 5-HT depleted were no significantly different from Bilateral L-allylglycine group.

To our knowledge, this is the first report of specific involvement of 5-HT transmission in mpFC in controlling immobility behavior in FST. Additional behavioral experiments showed that neither spontaneous locomotor activity nor emotional reactivity, measured through the plus maze and the open field test, were affected by selective 5-HT depletion (data not shown), thus ruling out a general motor impairment or an increase of anxiety-like behavior. Note that both control and 5-HT-depleted animals were unilaterally implanted with a microdialysis probe through which CSF was perfused. This was done to allow these experimental groups to be compared with those of subsequent experiments.

Selective 5-HT depletion in mpFC increases c-fos expression in BLA and reduces it in CeA following FST. To further confirm prefrontal 5-HT-amygdala connectivity, c-fos expression in ipsilateral amygdalar nuclei assessed following FST showed that depletion in mpFC significantly increased the $\mathrm{c}$-fos expression in BLA $\left(\mathrm{F}_{1.14}=11.25\right.$, $P<0.01$ ), whereas $c$-fos expression in CeA was significantly reduced in depleted mice in comparison with Sham animals $\left(\mathrm{F}_{1.14}=7.64, P<0.05\right)$ (Sham-treated group $(n=8), 5-\mathrm{HT}$ depleted group $(n=8)$ ) (Figure 4$)$.

A 5-HT prefrontal/GABA amygdala disconnection procedure reduced the immobility in FST. To assess the functional significance of this circuitry, a corticolimbic disconnection procedure was used that combined selective unilateral 5-HT depletion in the mpFC and infusion (by in vivo reverse microdialysis) of an inhibitor of GABA synthesis L-allylglycine in the controlateral BLA to produce functional GABA depletion, thereby disrupting prefrontalamygdalar serial connectivity bilaterally. Four groups of mice in the FST were tested: 1) unilateral mpFC 5-HTdepleted/controlateral BLA L-allylglycine; (2) unilateral mpFC 5-HT-depleted/controlateral BLA vehicle (CSF); (3) unilateral $\mathrm{mpFC}$ Sham/controlateral BLA L-allylglycine; and (4) unilateral mpFC Sham/controlateral BLA vehicle (CSF) (Figure 2). FST induced a clear-cut time-related increase of GABA output in BLA of mice receiving vehicle as shown by dialysate from mice having a probe in the BLA, whereas L-allylglycine caused GABA output to decrease dramatically throughout, as did selective 5-HT depletion in mpFC (see Figure 1). This response in mice receiving vehicle in BLA is consistent with that from restraint experiments showing a stress-induced GABA output increase. Unilateral selective mpFC 5-HT depletion in combination with a contralateral L-allylglycine infusion in BLA (disconnection) 

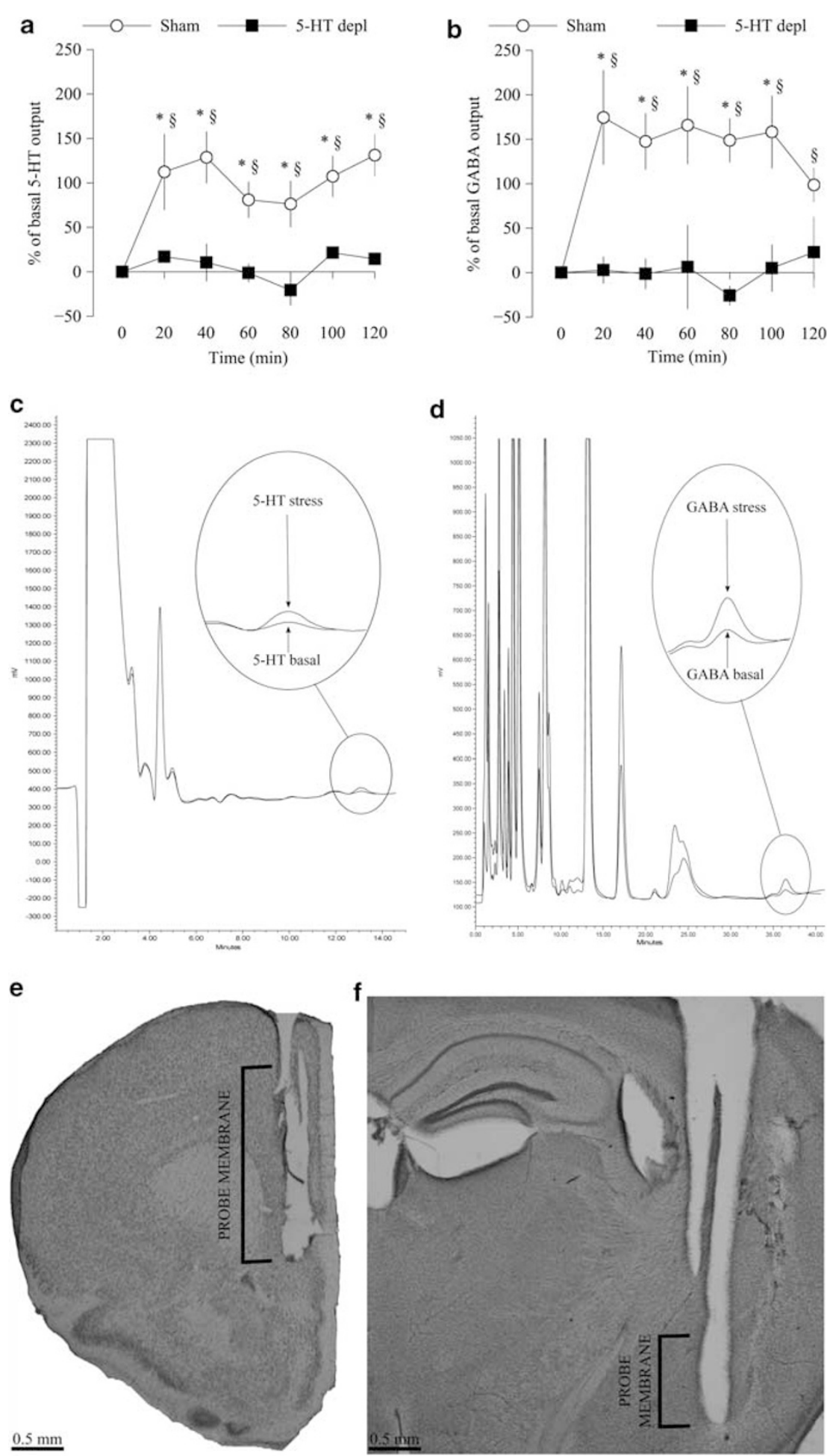

Figure 3 Effects of selective prefrontal cortical 5-hydroxytryptamine (5-HT) depletion on 5-HT outflow in the medial prefrontal cortex (mpFC) (a) and GABA outflow in the basolateral amygdala (BLA) (b) of mice exposed to 120 min of restraint. Results are expressed as percent changes (means \pm SE) from basal values. Statistical analyses were performed on raw data. $\S P<0.05$ from the basal values. ${ }^{*} P<0.05$ in comparison with the corresponding time point of sham group. Samples of chromatograms of basal and stress 5-HT outflow in the mpFC (c) and GABA outflow in the BLA (d). Representative positions of the probe in the mpFC (e) and BLA (f). The segment of the membrane probe is also shown.

caused a significant decrease in immobility behavior during the FST compared with three other control conditions $(\mathrm{F} 3,28=14,9, P<0.01$; Figure 5). (Disconnection $(n=8)$ $112.95 \pm 20.3 \mathrm{~s} ; \mathrm{mpFC}$ Sham unilateral/L-allylglycine in BLA controlateral $(n=8) 268.2 \pm 20.1 \mathrm{~s} ;$ mpFC 5-HT-depleted unilateral/CSF in BLA controlateral $(n=8) 263.8 \pm 31.4 \mathrm{~s}$; and mpFC Sham unilateral/CSF in BLA controlateral $(n=8)$ $297.9 \pm 7.4 \mathrm{~s}$.) Note that no significant differences were 

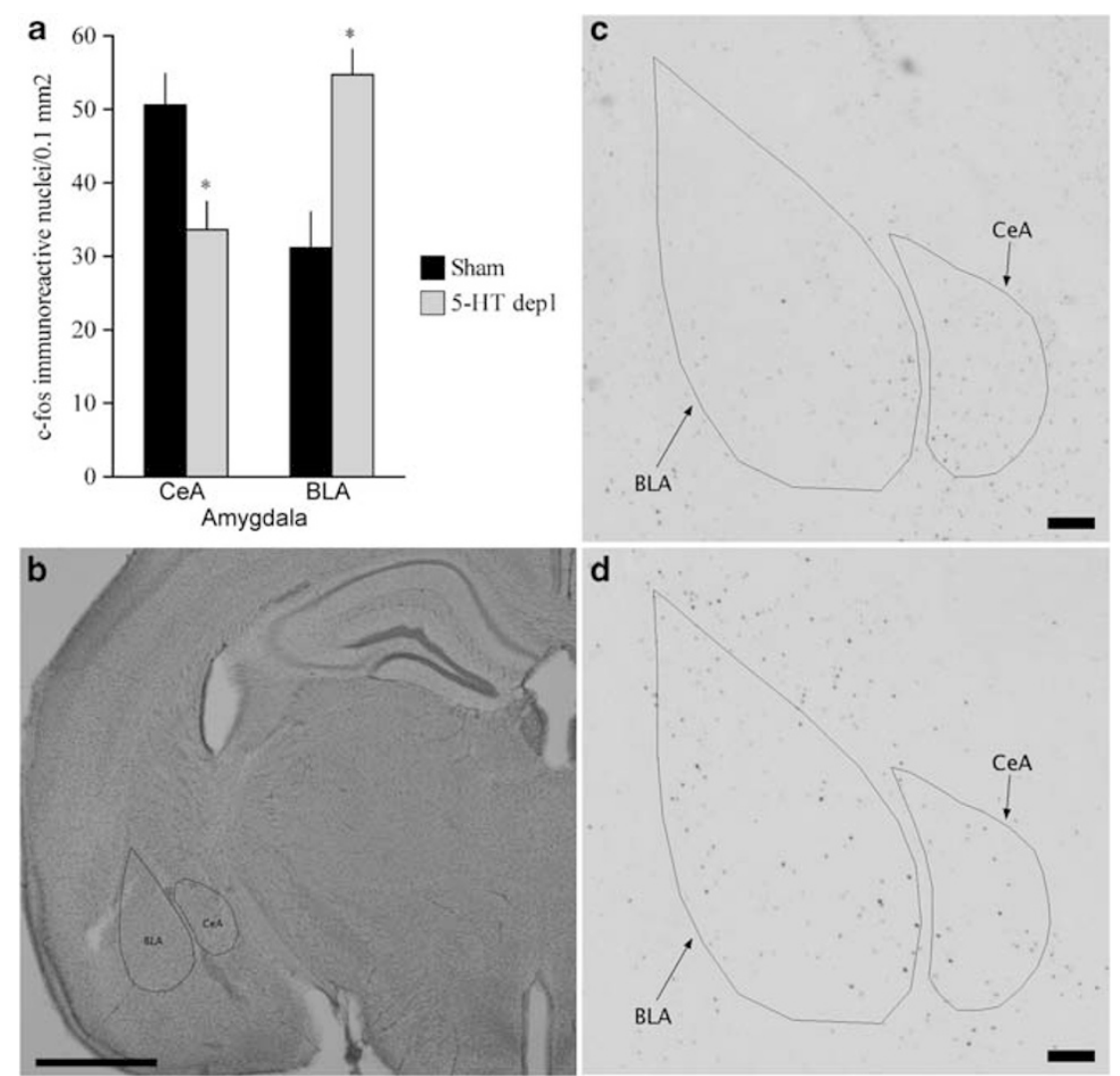

Figure 4 (a) Effects of selective prefrontal cortical 5-hydroxytryptamine (5-HT) depletion on Forced Swimming Test (FST)-induced c-fos immunoreactive cell nuclei (mean number of nuclei/0.I $\mathrm{mm}^{2} \pm \mathrm{SEM}$ ) in central amygdala (CeA) and basolateral amygdala (BLA). $* P<0.05$. (b) Representative Nissl-stained section illustrating CeA and BLA boundaries as revealed by cytoarchitecture. Scale bar $=1 \mathrm{~mm}$. Representative photomicrographs showing FST-induced c-fos immunoreactive cell nuclei in CeA and BLA of 5-HT-depleted group (c) and mpFC 5-HT sham group (d) (according to the atlas of Franklin and Paxinos, 1997). Scale bar $=100 \mu \mathrm{m}$.

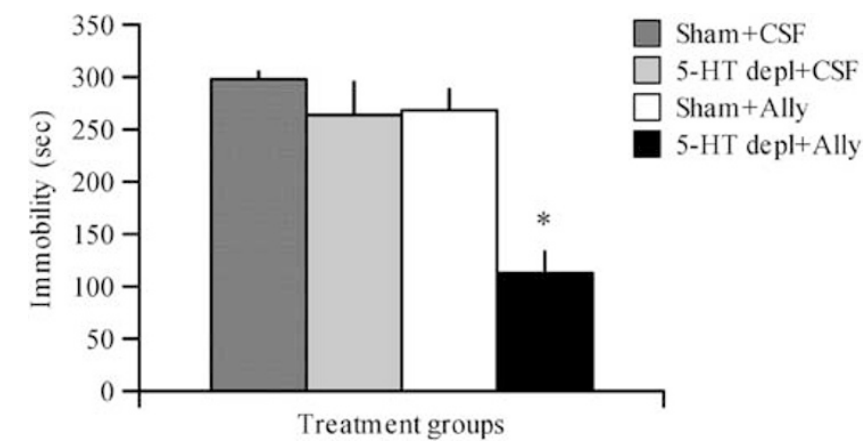

Figure 5 Effects of 5-hydroxytryptamine (5-HT) in medial prefrontal cortex (mpFC) and GABA in basolateral amygdala (BLA) disconnection on immobility in the Forced Swimming Test (FST). Results are expressed as mean $\pm \mathrm{SE}$ duration (second) of immobility. $* P<0.05$ in comparision with 5-HT sham + ally, 5-HT depl+cerebrospinal fluid (CSF), and 5-HT sham + CSF groups.

noted between the left and the right side of 5-HT depletion or L-allylglycine infusion treatment within each group on behavioral outcome. Moreover, immobility of animals having undergone unilateral mpFC 5-HT depletion and controlateral BLA L-allylglycine was not statistically different from immobility exhibited by mice 5-HT-depleted bilaterally in mpFC. These data show that 5-HT transmission, by controlling amygdala GABAergic transmission, modulates stress-related behavior in the FST.

\section{DISCUSSION}

Here evidence is reported of a brain system which, through modulation of GABA in the amygdala prefrontal cortical 5-HT, controls behavioral responses to stressful experiences sustaining immobility in the forced swimming paradigm that models depressive-like outcomes in rodents (Ebner et al, 2005, 2008; Singewald et al, 2011). It was first demonstrated that, in mice, a stressful experience such as restraint induces a time-dependent increase of 5-HT output in the mpFC and of GABA in the BLA, in agreement with previous reports (Reznikov et al, 2009; Pascucci et al, 2009), and that selective depletion of cortical 5-HT canceled out these stress-induced responses, thus pointing to a controlling role of GABAergic transmission in amygdala by prefrontal 5-HT during stress.

Electrophysiological studies indicated that previous stress and repeated administration of corticotrophin-releasing factor receptor agonist into the BLA reduces GABAergic neurotransmission in this structure, and this impairment is involved in the enhancement of conditioned fear behavior and anxiety (Rodríguez Manzanares et al, 2005; Rainnie et al, 2004). These results seems contrasting with ours showing that acute stress increases GABA release in BLA and that bilateral L-allylglycine infusion into the BLA reduces immobility during FST.

However, differences in experimental paradigms and procedures concerning pharmacological treatment, the time 
of analysis after stress, and the behavioral phenotype considered could account for differences between the above-cited studies and ours. Note that our results are in agreement with other microdialysis study showing that acute stress elicits an increase in GABA outflow in the BLA, whereas CeA outflow remained unchanged, and that prior repeated stress cancels out this response in BLA (Reznikov et al, 2009).

Recent findings suggest that IF and PL regions have different targets within the amygdala (Vertes, 2004; Gabbott et al, 2005). Specifically, the PL region sends projections to the BLA, whereas IF region sends projection to a region of the amygdala called the intercalated cell region (ITC). In our experiment, 5,7-DHT infusion in mpFC, although did not allow to target selectively these subregions, produced an about $84 \%$ reduction of $5-\mathrm{HT}$ tissue levels in PL region and about $68 \%$ reduction of 5-HT tissue levels in IL region, leaving unchanged amine levels in close areas. Thus, it is conceivable that the effect of 5-HT depletion in mpFC on GABA outflow in BLA involve principally the PL region.

To investigate if this neural connection in stress implies a brain system and to ascertain if such a system has a role in stress related behavior, animals were exposed to FST, which is a stressful condition allowing the individual to implement coping responses ranging from active to passive (depression-like). Moreover, we disrupted prefrontal/amygdalar serial connectivity bilaterally by means of a corticolimbic disconnection procedure that combined selective unilateral 5-HT depletion in the mpFC and pharmacological inactivation of GABA output through in vivo reverse microdialysis infusion of an inhibitor of GABA synthesis, L-allylglycine, in the controlateral BLA. Combining an otherwise ineffective unilateral infusion of L-allylglycine in the BLA of mice previously subjected to an ineffective unilateral 5-HT depletion resulted in a clear-cut decrease of immobility. Note that immobility was comparable to that exhibited by mice 5-HT depleted bilaterally in mpFC, and with that of mice receiving L-allylglycine bilaterally. These data indicate that the serial transfer of information between the mpFC and BLA guides behavioral response in the FST. Disconnection procedures involve unilateral manipulation of one structure in a neural system combined with manipulation of another intimately connected structure within the same system but on the opposite side of the brain, thereby disrupting the function of the serially interacting structures bilaterally. Thus, the disconnection manipulation should have behavioral effects very similar, if not identical, to those produced by bilateral manipulations of either structure alone within the system (Setlow et al, 2000; Floresco and Ghods-Sharifi, 2007; Belin and Everitt, 2008), but will have little or no effect if the structures are less directly, or serially, connected within a more diffuse neural system. The prefrontal-amygdala system envisaged here involves a parallel stress-induced increase of 5-HT and GABA transmission in the two brain regions, the former controlling the latter through neural pathways (circuitry) that can be validly hypothesized.

The critical role of prefrontal cortex-amygdala system in many behavioral phenotypes is acknowledged. The $\mathrm{mpFC}$ is involved in the integration and subsequent regulation of stimulus-driven amygdala response, via glutamatergic projections to population neurons within the amygdala
(Likhtik et al, 2005; Quirk et al, 2003), and different studies have demonstrated that aberration in this regulation leads to abnormal proccesing of emotional stimuli (Drevets, 1999).

The expression of 5-HT receptors on $\mathrm{mpFC}$ glutamatergic neurons suggests that their functional interactions may mediate 5 -HT effects on this circuit through top-down regulation of amygdala functioning (Amargos-Bosch et al, 2004).

Activation of 5-HT2a receptors in mpFC results in increased glutamatergic synaptic activity (Beique et al, 2007). Some authors have proposed that the excitatory action of this receptor 5-HT2a may be particularly prominent under conditions of high 5-HT release (Sharp et al, 2007), and a growing body of clinical psychopharmacological evidence is consistent with the hypothesis that blockade of 5-HT2a receptors might enhance the therapeutic effectiveness of SSRIs in depression patients (Marek et al, 2005). Thus, we speculate that high-level 5-HT release in $\mathrm{mpFC}$ under stress conditions could be able to activate the 5-HT2a receptor and consequently be responsible for expression of depressive-like behavior. The $\mathrm{mpFC}$ gates impulse transmission in amygdala from the BLA to CeA (Quirk et al, 2003). The CeA is a major output of the amygdala for the control of brain circuits involved in physiological and behavioral stress responses (Amaral et al, 1992). The activation of this nucleus was found to be associated to an increase in passive coping behavior (Stalnaker et al, 2009).

Thus, it can be assumed that stress-induced increase of 5-HT release in mpFC activates prefrontal cortical glutamatergic projection to the BLA, leading to increased GABA release within the BLA and to inhibition of CeA activity through the ITC (see below). This prefrontal-amygdala system may have a key role in depressive-like behavior.

Moreover, a neuronal circuit involving the projections from $\mathrm{mpFC}$ to the dorsal raphe nucleus may contribute to the regulation of amygdala reactivity through an indirect pathway. Indeed, it has been reported that the mpFC exerts an inhibitory influence on stress-induced activation of 5-HT neurons in the dorsal raphe nucleus (DRN) when stressful conditions are controllable (susceptible to coping efforts) by the organism (Amat et al, 2005), and 5-HT transmission in $\mathrm{mpFC}$ has a critical role in this control (Celada et al, 2002). Moreover, the DRN serotonergic system is a major modulator of the GABAergic system in the BLA, and dysregulation of this system has been envisaged in stress and mental disorders (Rainnie, 1999). Thus, it is likely that mpFC 5-HT regulates the amygdala GABAergic system through DRN 5-HT neurons (indirect pathway), which is consistent with a key role of the prefrontal-DRN system in coping and depressive-like behavior.

5-HT depletion in mpFC was found to significantly increase c-fos expression in the BLA and to reduce it in the CeA. These data indicate that $5-\mathrm{HT}$ release in $\mathrm{mpFC}$ modulates amygdala activation during the FST and that decreased immobility is linked respectively to increased and decreased c-fos expression in BLA and CeA. Although these changes of c-fos expression in BLA do not provide direct evidence of a GABA-dependent mechanism, it is conceivable that the changes in c-fos activation in this area depend on changes in GABA release. Indeed, it has been showed that repeated stress decreased expression of $\mathrm{c}-\mathrm{fos}$ in BLA 
interneuronal populations, and with specific regard to the GABA neuron population (Reznikov et al, 2008). Recent data from neuroimaging studies indicate that depression patients, who display a low-expressing HTT gene variant that has been seen to cause an increase of 5-HT release in mpFC (Mathews et al, 2004) in mice, are associated with functional abnormalities in the prefrontal cortex-amygdala circuit, specifically, amygdala hyperactivity coupled with hypofunction in the prefrontal cortex (Pezawas et al, 2005; Heinz et al, 2005). Moreover, it has been shown that active coping during an inescapable stress cause a decrease in c-fos in the CeA (Stalnaker et al, 2009). These results are consistent with those presented herein, because CeA activation was found to be associated to an increase of immobility, indicating that 5-HT transmission in mpFC has a role in this modulation, possibly by controlling BLA GABA release. Note that amygdalar ITC, which receive afferents from the BLA, could account for the present results. Indeed, ITC GABAergic neurons are an intraamygdala relay station exerting feed-forward inhibition of the CeA output (Pare et al, 2004). The CeA is a major output station in the amygdala, and its projections reach different structures in the brainstem, which have a role in generating behaviorally relevant outputs (LeDoux et al, 1988; Holstege et al, 1996). Finally, it has been demonstrated, that mpFC stimulation increases ITC c-Fos expression (Berretta et al, 2005) and produced an associated inhibition of CeA neurons (Quirk et al, 2003). Thus, we assume that 5-HT release in $\mathrm{mpFC}$ modulates BLA activation through stressinduced GABAergic transmission and consequently modulates the CeA activation through GABAergic ITC cell. In particular, reduced BLA GABA release, caused by $5-\mathrm{HT}$ depletion in mpFC, leads to activation of BLA nuclei. This activation could produce a decrease of $\mathrm{CeA}$ activation, through BLA GABA ITC neurons. The present results show that prefrontal cortical 5-HT transmission, through the control of GABAergic transmission in BLA, modulates immobilty in the FST. Substantial evidence indicates that the amygdala is involved both in depression and in the action of antidepressants (Krystal et al, 2002). Although depression is seen largely as a dysfunction in monoamine neurotransmission and all antidepressant strategies focus largely on monoamines, strong clinical and preclinical evidence implicates dysfunction of the GABAergic system in depression (Krystal et al, 2002). In rodents, the amygdala has been shown to be involved in depressive-like behavior in the FST, at least in part through GABA release (Ebner et al, 2005; Briones-Aranda et al, 2005). Different studies described antidepressant effects of the GABAb receptor agonist baclofen in FST (Cryan and Kaupmann, 2005), conceivably through presynaptic GABAb receptor activation leading to decrease of GABA release (Rea et al, 2005). In support of this hypothesis, we found that reduction of GABA release in the BLA reduced immobility in the FST, pointing to antidepressive effects of presynaptic GABAb autoreceptor stimulation in BLA. Substantial clinical and preclinical evidence implicates dysfunction of the GABA system in depression (Krystal et al, 2002; Sanacora, 2010), suggesting a complementary role of GABA and monoamines in mood disorders, and pointing to unbalanced multiple neurotransmitter systems in its etiology. The data presented herein present an intriguing opportunity in this sense, suggesting a role of serotonin and GABA neurotransmission in the prefrontal cortex-amygdala system in stress response and behavioral outcomes related to depression. This brain system suggests a way for developing therapeutic approaches aimed at modulating integrated functional mechanisms underlying adaptive/maladaptive behavior.

\section{ACKNOWLEDGEMENTS}

We thank Dr Sergio Papalia for his skillful assistance. This research was supported by 'Ricerca Corrente', Italian Ministry of Health and Ateneo 2011, Sapienza University of Rome.

\section{DISCLOSURE}

The authors declare no conflict of interest.

\section{REFERENCES}

Alcaro A, Cabib S, Ventura R, Puglisi-Allegra S (2002). Genotypeand experience-dependent susceptibility to depressive-like responses in the forced-swimming test. Psychopharmacology (Berl) 164: 138-143.

Amaral D, Price J, Pitkanen A, Carmichael T (1992). Anatomical organization of the primate amygdaloid complex. In Aggleton JP (ed) The Amygdala: Neurobiological Aspects of Emotion, Memory, and Mental Dysfuncton. Wiley-Liss: New York, pp 1-66.

Amargos-Bosch M, Bortolozzi A, Puig MV, Serrats J, Adell A, Celada $\mathrm{P}$ et al (2004). Co-expression and in vivo interaction of serotonin1 $\mathrm{A}$ and serotonin2 $\mathrm{A}$ receptors in pyramidal neurons of prefrontal cortex. Cereb Cortex 14: 281-299.

Amat J, Baratta MV, Paul E, Bland ST, Watkins LR, Maier SF (2005). Medial prefrontal cortex determines how stressor controllability affects behavior and dorsal raphe nucleus. Nat Neurosci 8: 365-371.

Anisman H, Irwin J, Beauchamp C, Zacharko R (1983). Crossstressor immunization against the behavioral deficits introduced by uncontrollable shock. Behav Neurosci 97: 452-461.

Beique JC, Imad M, Mladenovic L, Gingrich JA, Andrade R (2007). Mechanism of the 5-hydroxytryptamine $2 \mathrm{~A}$ receptor-mediated facilitation of synaptic activity in prefrontal cortex. Proc Natl Acad Sci USA 104: 9870-9875.

Belin D, Everitt BJ (2008). Cocaine seeking habits depend upon dopamine-dependent serial connectivity linking the ventral with the dorsal striatum. Neuron 57: 432-441.

Berretta S, Pantazopoulos H, Caldera M, Pantazopoulos P, Parz D (2005). Infralimbic cortex activation increases C-Fos expression in intercalated neurons of the amygdala. Neuroscience 132: 943-953.

Briones-Aranda A, Rocha L, Picazo O (2005). Alterations in GABAergic function following forced swimming stress. Pharmacol Biochem Behavior 80: 463-470.

Cabib S, Puglisi-Allegra S (1991). Genotype-dependent effects of chronic stress on apomorphine-induced alterations of striatal and mesolimbic dopamine metabolism. Brain Res 542: 91-96.

Cabib S, Puglisi-Allegra S (2012). The mesoaccumbens dopamine in coping with stress. Neurosci Biobehav Rev 36: 79-89.

Celada P, Puig MV, Mart'n-Ruiz R, Casanovas JM, Artigas F (2002). Control of the serotonergic system by the medial prefrontal cortex: potential role in the etiology of PTSD and depressive disorders. Neurotox Res 4: 409-419. 
Chowdhury GM, Fujioka T, Nakamura S (2000). Induction and adaptation of Fos expression in the rat brain by two types of acute restraint stress. Brain Res Bull 52: 171-182.

Conversi D, Orsini C, Colelli V, Cruciani F, Cabib S (2010). Association between striatal accumulation of FosB $/$ FosB and longterm psychomotor sensitization to amphetamine in mice depends on the genetic background. Behav Brain Res 217: $155-164$.

Cryan JF, Kaupmann K (2005). Don't worry 'B' happy!: a role for GABA(B) receptors in anxiety and depression. Trends Pharmacol Sci 26: 36-43.

Cullinan WE, Herman JP, Battaglia DF, Akil H, Watson SJ (1995). Pattern and time course of immediate early gene expression in rat brain following acute stress. Neuroscience 64: 477-505.

Day HE, Nebel S, Sasse S, Campeau S (2005). Inhibition of the central extended amygdala by loud noise and restraint stress. Eur J Neurosci 21: 441-454.

Drevets WC (1999). Prefrontal cortical-amygdalar metabolism in major depression. Ann N Y Acad Sci 877: 614-637 Review.

Ebner K, Bosch JO, Kromer SA, Singewald N, Neumann ID (2005). Release of oxytocin in the rat central amygdala modulates stresscoping behavior and the release of excitator amino acids. Neuropsychopharmacology 30: 223-230.

Ebner K, Singewald GM, Whittle N, Ferraguti F, Singewald N (2008). Neurokinin 1 receptor antagonism promotes active stress coping via enhanced septal 5-HT transmission. Neuropsychopharmacology 33: 1929-1941.

Fisher PM, Meltzer CC, Price JC, Coleman RL, Ziolko SK, Becker C et al (2009). Medial prefrontal cortex 5-HT(2A) density is correlated with amygdala reactivity, response habituation, and functional coupling. Cereb Cortex 19: 2499-2507.

Floresco SB, Ghods-Sharifi S (2007). Amygdala-prefrontal cortical circuitry regulates effort-based decision making. Cereb Cortex 17: 251-260.

Franklin KBJ, Paxinos G (1997). The Mouse Brain: In Stereotaxic Coordinates. Academic: San Diego.

Gabbott PL, Warner TA, Jays PR, Salway P, Busby SJ (2005). Prefrontal cortex in the rat: projections to subcortical autonomic, motor, and limbic centers. J Comp Neurol 492: 145-177.

Heinz A, Braus DF, Smolka MN, Wrase J, Puls I, Hermann D et al (2005). Amygdala-prefrontal coupling depends on a genetic variation of the serotonin transporter. Nat Neurosci 8: 20-21.

Holstege G, Bandler R, Saper CB (1996). The emotional motor system. Prog Brain Res 107: 3-6.

Hughes P, Dragunow M (1995). Induction of immediate early genes and the control of neurotransmitter-regulated gene expression within the nervous system. Pharmacol Rev 47: 133-178.

Koolhaas JM, De Boer SF, Buwalda B, Van Reenen K (2007). Individual variation in coping with stress: a multidimensional approach of ultimate and proximate mechanisms. Brain Behav Evol 70: 218-226.

Krystal JH, Sanacora G, Blumberg H, Anand A, Charney DS, Marek $\mathrm{G}$ et al (2002). Glutamate and GABA systems as targets for novel antidepressant and moodstabilizing treatments. Mol Psychiatry 1: 71-80.

Lazarus R (1966). Psychosocial Stress and the Coping Process. McGraw-Hill: New York.

LeDoux JE, Iwata J, Cicchetti P, Reis DJ (1988). Different projections of the central amygdaloid nucleus mediate autonomic and behavioral correlates of conditioned fear. J Neurosci 8: 2517-2529.

Likhtik E, Pelletier JG, Paz R, Pare D (2005). Prefrontal control of the amygdala. J Neurosci 25: 7429-7437.

Maier SF, Seligman ME (1976). Learned helplessness: theory and evidence. J Exp Psychol 105: 3-46.

Maier SF, Watkins LR (2010). Role of the medial prefrontal cortex in coping and resilience. Brain Res 1355: 52-60.
Marek GJ, Martin-Ruiz R, Abo A, Artigas F (2005). The selective 5- HT2A receptor antagonist M100907 enhances antidepressant-like behavioral effects of the SSRI fluoxetine. Neuropsychopharmacology 30: 2205-2215.

Martijena ID, Rodriguez Manzanares PA, Lacerra C, Molina VA (2002). Gabaergic modulation of the stress response in frontal cortex and amygdala. Synapse 45: 86-94.

Mathews TA, Fedele DE, Coppelli FM, Avila AM, Murphy DL, Andrews AM (2004). Gene dose-dependent alterations in extraneuronal serotonin but not dopamine in mice with reduced serotonin transporter expression. J Neurosci Methods 140: $169-181$.

McEwen BS (2001). Plasticity of the hippocampus: adaptation to chronic stress and allostatic load. Ann N Y Acad Sci 933: 265-277.

McEwen BS (2007). Physiology and neurobiology of stress and adaptation: central role of the brain. Physiol. Rev 87: 873-904.

Pare D, Quirk GJ, LeDoux JE (2004). New vistas on amygdala networks in conditioned fear. J Neurophysiol 92: 1-9.

Pascucci T, Andolina D, La Mela I, Conversi D, Latagliata C, Ventura R et al (2009). 5- Hydroxytryptophan rescues serotonin response to stress in prefrontal cortex of hyperphenylalaninaemic mice. Int J Neuropsychopharmacol 14: 479-489.

Pezawas L, Meyer-Lindenberg A, Drabant EM, Verchinski BA, Munoz KE, Kolachana BS et al (2005). 5-HTTLPR polymorphism impacts human cingulate-amygdala interactions: a genetic susceptibility mechanism for depression. Nat Neurosci 8: 828-834.

Porsolt RD, Le Pichon M, Jalfre M (1977). Depression: a new animal model sensitive to antidepressant treatments. Nature 266: 730-732.

Puglisi-Allegra S, Cabib S, Pascucci T, Ventura R, Cali F, Romano $\mathrm{V}$ (2000). Dramatic brain aminergic deficits in a genetic mouse model of phenyketonuria. Neuroreport 11: 1361-1364.

Quirk G-J, Likhtik E, Pelletier J-G, Pare D (2003). Stimulation of medial prefrontal cortex decreases the responsiveness of central amygdala output neurons. J Neurosci 23: 8800-8807.

Rainnie DG (1999). Serotonergic modulation of neurotransmission in the rat basolateral amygdala. J Neurophysiol 82: 69-85.

Rainnie DG, Bergeron R, Sajdyk TJ, Patil M, Gehlert DR, Shekhar A (2004). Corticotrophin releasing factor induced synaptic plasticity in the amygdala translates stress into emotional disorders. J Neurosci 24: 3471-3479.

Rea K, Cremers TI, Westerink BH (2005). HPLC conditions are critical for the detection of GABA by microdialysis. J Neurochem 94: 672-679.

Reznikov LR, Reagan LP, Fadel JR (2008). Activation of phenotypically distinct neuronal subpopulations in the anterior subdivision of the rat basolateral amygdala following acute and repeated stress. J Comp Neurol 508: 458-472.

Reznikov LR, Reagan LP, Fadel JR (2009). Effects of acute and repeated restraint stress on GABA efflux in the rat basolateral and central amygdala. Brain Res 1256: 61-68.

Rodríguez Manzanares PA, Isoardi NA, Carrer HF, Molina VA (2005). Previous stress facilitates fear memory, attenuates GABAergic inhibition, and increases synaptic plasticity in the rat basolateral amygdala. J Neurosci 25: 8725-8734.

Sanacora G (2010). Cortical inhibition, gamma-aminobutyric acid, and major depression: there is plenty of smoke but is there fire? Biol Psychiatry 57: 397-398.

Setlow B, Roozendaal B, McGaugh JL (2000). Involvement of a basolateral amygdala complex-nucleus accumbens pathway in glucocorticoid-induced modulation of memory consolidation. Eur J Neurosci 12: 367-375.

Sharp T, Boothman L, Raley J, Queree P (2007). Important messages in the 'post': recent discoveries in 5-HT neuron feedback control. Trends Pharmacol. Sci 28: 629-636. 
Singewald GM, Rjabokon A, Singewald N, Ebner K (2011). The modulatory role of the lateral septum on neuroendocrine and behavioral stress responses. Neuropsychopharmacology 36: 793-804.

Stalnaker TA, Espana RA, Berridge CW (2009). Coping behavior causes asymmetric changes in neuronal activation in the prefrontal cortex and amygdala. Synapse 63: 82-85.

Taylor AE, Stanton AL (2007). Coping resources, coping processes, and mental health. Annu Rev Clin Psychol 3: 377-401.

Ursin H, Olff M (1995). Aggression, defense, and coping in humans. Aggressive Behavior 21: 13-19.

Valentino RJ, Lucki I, Van Bockstaele E (2010). Corticotropinreleasing factor in the dorsal raphe nucleus: Linking stress coping and addiction. Brain Res 1314: 29-37.
Ventura R, Morrone C, Puglisi-Allegra S (2007). Prefrontal/ accumbal catecholamine system determines motivational salience attribution to both reward- and aversion-related stimuli. Proc Natl Acad Sci USA 104: 5181-5186.

Vertes RP (2004). Differential projections of the infralimbic and prelimbic cortex in the rat. Synapse 51: 32-58.

Wechsler B (1995). Coping and coping strategies: a behavioural view. Appl Anim Behaviour Sci 43: 123-134.

Wellman CL, Izquierdo A, Garrett JE, Martin KP, Carroll J, Millstein $\mathrm{R}$ et al (2007). Impaired stress-coping and fear extinction and abnormal corticolimbic morphology in serotonin transporter knock-out mice. J Neurosci 27: 684-691. 